\title{
ON LAX PAIRS AND MATRIX EXTENDED SIMPLE TODA SYSTEMS
}

\author{
M. LEGARÉ
}

Received 17 November 2004 and in revised form 17 June 2005

A $A_{1}$ Toda system is extended via Lax pair formulations in order to probe noncommutative variables extensions. Systems, some solvable, are built using matrix generalizations.

\section{Introduction}

Noncommutative theories have been studied and probed from different viewpoints (see reviews $[18,34,48])$. For instance, a number of noncommutative generalizations of integrable systems were presented (see, e.g., $[9,16,17,24,39])$. Solutions were investigated using the dressing method and Riemann-Hilbert problems, formulations, and properties such as infinite sets of conserved quantities were shown, and linear systems (or Lax pairs) were exhibited in different articles (e.g., [16, 17, 23, 30, 39, 40, 41]). Systems such as solitons, instantons, monopoles, Yang-Mills-Higgs, and nonlinear sigma models in $2+1$ dimensions have been explored with noncommutative variables, raising certain similarities (see, e.g., $[22,29])$.

Noncommutative (Yang-Mills) theories have been linked to string theories with nontrivial B-field. It is known that self-dual Yang-Mills equations in 4-dimensional space (or their generalizations) lead, through reductions, to many integrable systems in lower dimensions, and for this, they have also been initially labelled as "master equations" [52]. Similarly, supersymmetric integrable systems in dimensions smaller than 4 have also been found to have a reduction relation with respect to supersymmetric self-dual Yang-Mills equations. As a first step towards a (possible) noncommutative "master system," it has then been mentioned that a noncommutative version of self-dual supersymmetric YangMills systems could provide via reductions noncommutative generalizations of (supersymmetric) integrable systems [35].

In the following, our general interest is twofold: noncommutativity and deformations. Noncommutativity can be introduced using different structures, as shown in [33]. A basic noncommutativity of variables could be imposed through $\left[x^{\mu}, x^{\nu}\right]=i \theta^{\mu \nu}$, and can be associated to a $*$-product. Use of these noncommutative variables (or $*$-product) could also be seen as probing deformations of (integrable) systems, with a deformation 
parameter $(\theta)$. Other noncommutative structures could as well be involved, such as a matrix spaces, where elements could, to a certain extent, be interpreted as parameters of deformations. Matrix structures occurred at a different level with (D0) branes, where spatial coordinates of a system of such branes become (noncommuting) matrices $[4,53]$, and more generally with the consideration of transverse coordinates (these aspects are related to matrix theory [4]). A shift to such (noncommutative) structures could be adopted for simple systems to probe their deformations and integrability. Among integrable systems, the Toda models $[37,49]$ have been the objects of various (integrable) generalizations. For instance, an integrable isospectral deformation of an arbitrary $N \times N$ real elements matrix, which is related to a generalization of the nonperiodic Toda lattice, has been obtained [26], and integrable generalizations of the Toda chains have been formulated with $\mathbb{Z}$-gradations of classical Lie algebras using a Lax formulation [54], non-Abelian versions of Toda models have been written (see $[19,20,43]$ and references therein) as well as supersymmetric versions [36] and Toda-like systems [11].

In this short communication, generalizations of the simple $\left(A_{1}\right)$ Toda system $[37,43]$, which is a system with 2 particles, is considered and probed at different levels. First as examples, simple "toy" extensions of the Lax pairs associated to the $A_{1}$-model are presented, along with solutions, by varying the time evolution part (or auxiliary matrix) of the Lax system (or pair). Quantization would lead to a noncommutative structure and can be studied independently. Then, in the spirit of the Moyal-Nahm equations [3] and the (noncommutative) Toda field equations in $[15,31]$, a Moyal $*$-product is introduced in the matrix Lax equations. The generalized Toda systems presented at the beginning of this paper are left unchanged by this modification, but a method of solution for various generalizations is discussed within the context of the Weyl correspondence.

Pursuing generalizations with noncommutative behaviour and some type of deformations, noncommutative variables such as matrices are subsequently introduced in the equations of interest. These matrix extensions, which also preserve the coordinates splitting, are derived using a Lax pair formulation, extending formulations by [27], then, for instance, one could examine their relation to integrability (spectral curves, etc.). A noncommutative aspect is also realized through a Hamiltonian formulation, and similarly integrability could be investigated there. But finally, an extension of the time evolution part or auxiliary part of the Lax pair will be dictated by a new matrix, this in analogy with the $A_{1}$-model toy extensions via auxiliary matrices mentioned above. Let us note that $R$ hierarchies can be generated in this manner (see, e.g., [47]), and that integrable systems and deformations have been retrieved and generated in such a setting $[8,42,45,50,51]$. The classification of $R$-operators for simple Lie algebras would provide a good set of such (time-evolution) auxiliary matrices. It gives rise to a model building which could explore aspects of integrability, noncommutativity, and deformations using matrices. In the last section, examples of matrix substitutions to probe certain of these aspects are presented using trivial and nontrivial $R$-operators, where certain systems are solvable. Finally, a discussion of the results and further generalizations conclude this short communication, with its main objective to present certain (to our knowledge new) examples of extensions with noncommutativity and deformations. 


\section{Generalized auxiliary matrix and $A_{1}$ Toda model}

A well-known Lax pair formulation of the one-dimensional harmonic oscillator (of unit frequency) is simply given by [21]

$$
L=\left[\begin{array}{cc}
p(t) & q(t) \\
q(t) & -p(t)
\end{array}\right], \quad M=\frac{1}{2}\left[\begin{array}{cc}
0 & -1 \\
1 & 0
\end{array}\right],
$$

with $\dot{L}=[L, M]$.

Generalizations can be obtained by allowing the time evolution associated to the $M$ auxiliary matrix to take the form [21]

$$
M=\frac{1}{2}\left[\begin{array}{cc}
0 & -f(q, p, t) \\
f(q, p, t) & 0
\end{array}\right],
$$

where $f$ is an arbitrary function of $q, p$, and $t$. Still in all cases, $\operatorname{tr}\left(L^{2}\right)=2\left(p^{2}+q^{2}\right)$ is a constant of motion, and these systems are integrable for $f(q, p)$. For specific functions $f$, they can be seen as "solvable" (in the sense of [10]). It is known that for $f(q, p, t)=q$, the nonperiodic or open $A_{1}$ Toda system (for 2 particles) is retrieved, in usual form with $q=e^{\phi}$. A Lax pair with spectral parameter $\lambda$ for these Toda systems is given by [27]

$$
L=\left[\begin{array}{cc}
p_{1} & q_{1} \\
q_{1}+\lambda q_{2} & p_{2}
\end{array}\right], \quad M=\frac{1}{2}\left[\begin{array}{cc}
0 & -q_{1} \\
q_{1}-\lambda q_{2} & 0
\end{array}\right],
$$

where $p_{1}+p_{2}=0, q_{1} q_{2}=1$.

If, in the original Lax pair (with auxiliary matrix (2.2)), one allows $f=q^{n}$, where $n \in \mathbb{Z}$, then the Lax equation leads to an ordinary differential equation

$$
\ddot{\phi}+(1-n)(\dot{\phi})^{2}+e^{2 n \phi}=0,
$$

with a "velocity-dependent" nonlinear term $(\dot{\phi})$, where solutions are given with simple integrations

$$
\int_{q_{0}}^{q} \frac{d \tilde{q}}{\tilde{q}^{n} \sqrt{C-\tilde{q}^{2}}}= \pm\left(t-t_{0}\right)
$$

with the invariant $C=p^{2}+q^{2}$ (see, e.g., [25] using Bernoulli differential equation).

The compatibility condition for the linear system

$$
\dot{\psi}=-M \psi, \quad L \psi=E \psi,
$$

where $\psi$ is a function of $q, p, t$, gives rise to the previously mentioned systems generalized from the (commutative) $A_{1}$ Toda model. Some form of noncommutativity along the above-mentioned $\theta$ basic structure could be introduced via quantization. From the Lagrangian $L(q, \dot{q})=1 / 2\left[(\dot{q})^{2} / q^{2 n}-q^{2}\right]$, one deduces the Hamiltonian $H(q, p)=1 / 2\left(p^{2} q^{2 n}+\right.$ $q^{2}$ ), with the canonical symplectic form $\omega=d p \wedge d q$, to obtain the above $q$ equations (2.5), which can as well be derived from the Hamilton equations for the Hamiltonian 
2738 On Lax pairs and matrix extended simple Toda systems

$\tilde{H}(q, p)=-1 / 2\left(q^{2}+p^{2}\right)$, with $\tilde{\omega}=\left(1 / q^{n}\right) d p \wedge d q$. A quantization might be of interest via a suitable quantum $H(q, p)$. Studies of quantization have already been carried out for $n=1$ with $q=e^{\phi}$ (see $[38,44]$ and references therein).

\section{Moyal or noncommutative Lax equations}

Versions of the Lax equations with the insertion of the $*$-product in a pseudodifferential operator setting (e.g., $[12,28,46]$ ) or in a matrix formulation have been presented. Moreover, the (non-Abelian) Toda field equations can in fact be generalized to a noncommutative version with the $*$-product using a matrix Lax pair $[15,31]$. But in the latter situation, the noncommutativity between the two variables disappears with onedimensional translational reductions. However, a noncommutativity could be introduced for the phase space variables, as it was done for the Moyal-Nahm equations in [3].

Let $q, p$ denote the phase space variables, and let us define the product $*$ as follows between two functions $f$ and $g$ on phase space:

$$
f(q, p) * g(q, p)=f(q, p) e^{i \theta\left(\overleftarrow{\partial}_{q} \vec{\partial}_{p}-\overleftarrow{\partial}_{p} \vec{\partial}_{q}\right)} g(q, p)
$$

where $\theta$ is a noncommutative or deformation parameter. One can then define an $(n \times n)$ matrix Lax pair $L(q, p, t), M(q, p, t)$ with noncommutative Lax equation

$$
\dot{L}=[L, M]=L * M-M * L,
$$

where the matrix $*$-product between two $(n \times n)$ matrices $A$ and $B$ is given by $(A * B)_{i j}=$ $\sum_{k} A_{i k} * B_{k j}$, with $i, j, k=1, \ldots, n$. A solution could be written as

$$
M=-\dot{g} * g^{-1}, \quad L=g * L_{0} * g^{-1},
$$

where $g * g^{-1}=\mathbf{1}_{n}$, with $g=g(q, p, t)$. These Lax equations could be seen as compatibility conditions of a $*$-extended linear system $L * \psi=E * \psi, \dot{\psi}=-M * \psi$, where $\dot{E}=0$ and $\psi=\psi(q, p, t)$ are $n$-column vectors. The Lax equations still have a gauge invariance:

$$
\tilde{L}=h * L * h^{-1}, \quad \tilde{M}=h * M * h^{-1}-\dot{h} *\left(h^{-1}\right),
$$

where $h * h^{-1}=\mathbf{1}_{n}$, but $\operatorname{tr}\left(L^{m}\right)$, where $m$ is a positive integer are not necessarily constants of motion.

For the above auxiliary $M$ matrix generalizations of the $A_{1}$-model, the Lax equations lead to

$$
\dot{q}=-\frac{1}{2}\left(q^{n} * p+p * q^{n}\right), \quad \dot{p}=q^{n+1},
$$

which reduce to the known commutative equations. Let us comment that for generalizations with various functions of $q, p, t$ in the Lax matrix $L$ and its auxiliary matrix $M$ in (3.2), one can use the Weyl correspondence. Since for any element of $L$, denoted $L_{i j}$,

$$
\frac{d L_{i j}}{d t}=\frac{\partial L_{i j}}{\partial t}+\frac{\partial L_{i j}}{\partial q} \dot{q}+\frac{\partial L_{i j}}{\partial p} \dot{p}=\frac{\partial L_{i j}}{\partial t}+\left\{H, L_{i j}\right\}_{\text {P. B. }}
$$


where $H$ is a suitable Hamiltonian such that the Hamilton equations of motion are $\dot{q}=$

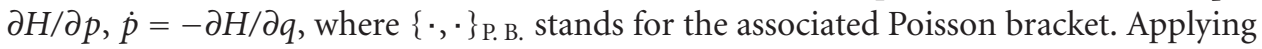
the inverse Weyl transformation $\left(W^{-1}\right)$, one can derive $[13,14]$ the following relation:

$$
\frac{\partial \widehat{L}}{\partial t}+\mathcal{W}^{-1}\left[\{H, L\}_{P . B .}\right]=[\widehat{L}, \widehat{M}],
$$

with $\mathscr{W}\left[\widehat{L}_{i j}\right]=L_{i j}, \mathcal{W}\left[\widehat{M}_{i j}\right]=M_{i j}$ where $\widehat{L}_{i j}, \widehat{M}_{i j}$ are operators on a Hilbert space, the element $i j$ of $\hat{L}$ being denoted by $\widehat{L}_{i j}$. One notes that

$$
W\left[-\frac{i}{2 \theta}[\hat{H}, \widehat{L}]\right]=\frac{1}{\theta} H \sin \left(\theta\left(\overleftarrow{\partial}_{q} \vec{\partial}_{p}-\overleftarrow{\partial}_{p} \vec{\partial}_{q}\right)\right) L
$$

which could be useful for small $\theta$ approximations. Many generalizations can be considered, but a focus on different aspects, thought to be of more interest, follows.

\section{Matrix generalizations of the $A_{1}$-Toda model}

Since the above simple $*$-product generalizations of the Lax equations lead to known equations, and since $(L \dot{\otimes} \mathbf{1})=[L \otimes \mathbf{1}, M \otimes 1]$, with the unit matrix 1 , is equivalent to the previous ordinary (non-Moyal) Lax equations, one could explore extensions involving noncommutative objects such as matrices, in the spirit of D0 branes. Isospectral deformations related to nonperiodic Toda lattices have been studied by [26], with $\dot{L}=[M, L]$, where $M=L_{>0}-L_{<0}$, with strictly upper $(>0)$ and lower $(<0)$ triangular parts of $L$. Different avenues are explored in what follows. First, a Hamiltonian description can be attempted.

\section{Matrix Hamiltonian generalizations}

Let $q=e^{\phi}$, and let $p, \phi$ be Hermitian $\left(p^{\dagger}=p\right.$ and $\phi^{\dagger}=\phi$, or $\left.\phi, p \in u(n)\right)$, where $u(n)$ is the Lie algebra of the unitary group $U(n)$. Let us define a Hamiltonian

$$
H(q, p)=\frac{1}{2} \operatorname{tr}\left(p^{2}+e^{(2 \phi)}\right)
$$

and a canonical symplectic form $\omega=\sum_{i, j} d p_{i j} \wedge d \phi_{j i}$. The Hamilton equations are consequently

$$
\dot{\phi}_{i j}=\frac{\partial H}{\partial p_{j i}}=p_{i j}, \quad \dot{p}_{i j}=-\frac{\partial H}{\partial \phi_{j i}}=-\left(e^{2 \phi}\right)_{i j},
$$

leading to the coupled ordinary differential equations

$$
\ddot{\phi}_{i j}=-\left(e^{2 \phi}\right)_{i j}
$$

for $i, j=1, \ldots, n$. This system is not known to be completely integrable for $n \neq 1$ positive integer values, but it provides a simple noncommutativity of the variables. 


\section{Lax equations generalizations}

Instead of working with the $\phi$ variable, we here implement the matrix generalization at the level of the variable $q$. A straightforward generalization uses the Lie algebra $\operatorname{sl}(2, \mathbb{R})$ for $L$ and $M$ matrices in the following manner:

$$
L=\left[\begin{array}{cc}
P(t) & Q(t) \zeta \\
Q(t) \zeta^{-1} & -P(t)
\end{array}\right], \quad M=\frac{1}{2}\left[\begin{array}{cc}
0 & -Q(t) \zeta \\
Q(t) \zeta^{-1} & 0
\end{array}\right],
$$

where $\zeta$ is a parameter and $P^{\dagger}=P, Q^{\dagger}=Q$, that is $Q, P \in u(n)$ again. The Lax equations $\dot{L}=[L, M]$ then lead to a noncommutative extension when $n>1$ integer of the $A_{1}$ Toda equations

$$
\dot{P}=Q^{2}, \quad \dot{Q}=-\frac{1}{2}(P Q+Q P)
$$

$L$ and $M$ can be seen as valued in $u(n) \otimes \operatorname{sl}(2, \mathbb{R}) \otimes \mathbb{C}$. Note that $\operatorname{tr}\left(L^{2}\right)=2 \operatorname{tr}\left(P^{2}+Q^{2}\right)$ is a nontrivial invariant, as well as $\operatorname{tr}\left(L^{4}\right)$, but the expressions $\operatorname{tr}\left(L^{2 m+1}\right)$, where $m$ is a nonnegative integer, vanish. The above generalization lacks the presence of the parameter $\zeta$ in the characteristic equation $\operatorname{det}\left(L-E \mathbf{1}_{n}\right)=0$.

Another formulation close to the Lax equations (6.1) presented previously involves $n \times n$ matrices on $\mathbb{R}: p_{1}, p_{2}$, and $q_{1}, q_{2}$, which both belong to the Lie group $\operatorname{GL}(n, \mathbb{R})$ (matrix fundamental representations of GL, $\mathrm{gl}$ and $\mathrm{sl}$ are used in the following). The matrices $L$ and $M$ belong to $\operatorname{sl}(2 n, \mathbb{R})$, with the constraints $q_{2}=q_{1}^{-1}, p_{1}+p_{2}=0$, and are given by (2.3) with the substitution of matrix valued elements. Their Lax equations can be written as

$$
\dot{p}_{1}=q_{1}^{2}, \quad \dot{q}_{1}=-\frac{1}{2}\left(q_{1} p_{1}+p_{1} q_{1}\right)
$$

It is found that $\operatorname{tr}\left(L^{2}\right)=2 \operatorname{tr}\left(p_{1}^{2}+q_{1}^{2}+\lambda \mathbf{1}_{n}\right), \operatorname{tr}\left(L^{3}\right)=0$, and that $\operatorname{tr}\left(L^{4}\right)$ includes terms such as $\operatorname{tr}\left(\left(p_{1}^{2}+q_{1}^{2}\right)^{2}-\left(q_{1} p_{1}-p_{1} q_{1}\right)^{2}\right)$ and $\operatorname{tr}\left(2 q_{1} p_{1} q_{1}^{-1} p_{1}-p_{1}^{2}\right)$. For $n=2$ or $L \in \operatorname{sl}(4, \mathbb{C})$, the spectral curve $\operatorname{det}\left(L-E \mathbf{1}_{4}\right)=0$ leads to a vanishing polynomial $P\left(E^{4}, E^{2}, \lambda, \lambda^{2}\right)=0$, as characteristic curve, which confirms that there is not enough constants of motion found via the traces of powers of $L$ to justify the complete integrability of this system. The corresponding algebraic curve is closer to a hyperelliptic one, and certain singularities might be avoided for certain sets of (matrix elements) coefficients. Eigenvalues (e.g., bundles on (part of) the spectral curves) can be considered for the Lax systems described. The $N$ particles $A_{N-1}$ Toda Lax equations could as well be extended using the Lax pairs in [27], using the substitution of the $p_{i}$ 's and $q_{i}$ 's with matrices. It is noted that the general open $A_{N-1}$ Toda system has been handled in [27] (with (reducible singular) algebraic curves).

\section{7. $R$-operators and matrix Lax equations extensions}

In a manner similar to the introductory considerations in Section 2 on generalizations of time evolution of the Lax pair associated to nonperiodic Toda systems, matrix extensions of the $A_{1}$ Toda model will rely as well on generalizations of the auxiliary matrix $M$ (which could be seen as deformations) through an $R$-operator. It can also be recalled that 
the $R$-operator can lead to a set of hierarchies once given a set of invariants based on $L$. The Lax equations obtained through these generalizations still would have to be probed independently for consistency and nontrivial solutions, but a classification of $R$-operators provides a large range of possible extensions of the auxiliary matrix $M$, and thus possible nontrivial systems.

In order to set our notation, let us introduce a Lie algebra $\mathscr{G}$, which in the following will be either a semisimple Lie algebra or the Lie algebra $\operatorname{gl}(n, \mathbb{R})$, with its dual denoted $\mathscr{G}^{*}$.

An $R$-operator is obtained when the linear mapping $R: G \rightarrow G$ gives rise to a new Lie algebra, denoted $\mathscr{G}_{R}$, via the bracket

$$
[X, Y]_{R}=[R(X), Y]+[X, R(Y)]
$$

It brings a new Lie-Poisson bracket $\{\cdot, \cdot\}_{R}$ on $G^{*}$ [47]. It is also known that $R$ satisfying the modified Yang-Baxter equations leads to such new Lie-Poisson bracket structure. If $R$ is equal to half the identity isomorphism on $\mathscr{G}$, then the usual Lie algebra bracket is retrieved.

For a Hamiltonian $H$ belonging to the set of smooth functions on $\mathscr{G}^{*}$, which is a coadjoint invariant of the algebra $\mathscr{G}$, the Hamilton equations of motion, given a scalar product $(\langle\cdot, \cdot\rangle)$ nondegenerate and invariant (i.e., invariant if $\langle X,[Y, Z]\rangle=\langle[X, Y], Z\rangle$, for any $X, Y, Z \in \mathscr{G})$, can be given in $\operatorname{Lax}$ form $\dot{L}=[L, M],[2,43,47]$ where

$$
M=R(\nabla H(L))
$$

with coadjoint invariants in involution with respect to the new Poisson bracket $\left(\{\cdot, \cdot\}_{R}\right)$. Let us recall [47] that a structure of Poisson submanifold of the Poisson manifold $\mathscr{G}$ associated with the Poisson bracket $\{\cdot, \cdot\}_{R}$ is desired to write an $r$-matrix interpretation of the latter Lax equations with $M$ as in (7.2). A bi-Hamiltonian structure could lead to further invariants in involution [47]. Many $R$-operators arise from the direct sum splitting of $\mathscr{G}_{=}=\mathscr{G}_{+} \oplus \mathscr{G}_{-}$, as vector space, of two subalgebras $\mathscr{G}_{+}, \mathscr{G}_{-}$of $\mathscr{G}_{\text {, with factorization for }}$ solutions $[2,43,47]$.

One could also consider generalizations of the equations of motion based on quadratic $r$-matrices, such as those leading to Sklyanin brackets, where, for instance, the latter can be obtained for skew-symmetric $R$-operators obeying the modified Yang-Baxter equations (let us mention that cubic structures could also be formulated with corresponding Lax equations, see, e.g., [32]). In the quadratic case, given a coadjoint invariant Hamiltonian $H(L)$, the Hamilton equations of motion have the usual Lax form $\dot{L}=[L, M]$, where [47]

$$
M=R(L \cdot \nabla H(L))
$$

provided $G$ has a multiplication $(\cdot)$ with Lie bracket $[X, Y]=X \cdot Y-Y \cdot X$, and is equipped with a bi-invariant scalar product $\langle X, Y \cdot Z\rangle=\langle X \cdot Y, Z\rangle=\langle Z \cdot X, Y\rangle$. This is satisfied for the Lie algebra $G=\operatorname{gl}(n, \mathbb{R})$, with the matrix multiplication in its $n \times n$ matrix representation endowed with the trace $(\operatorname{tr})$ as scalar product. More general $R$-operators related to quadratic structures can be used. 
Certains types of $R$-operators generalizations have previously been carried out (see $[8,51]$ and references therein). For example, generalizations using $R$-operators to generate Toda-like integrable systems have been discussed in [50], more recently Toda type discrete hierachies have been examined with $R$-matrix solutions of the modified YangBaxter equations [1].

As an example of this type of model building with respect to matrix generalizations of Toda sytems, let $L \in \operatorname{sl}(4, \mathbb{R})$ be of the form

$$
L=\left[\begin{array}{cc}
p_{1} & q_{1} \\
q_{1} & -p_{1}
\end{array}\right]
$$

where the matrices $q_{1}$ and $p_{1}$ are both $2 \times 2$ matrices on $\mathbb{R}$, and are expressed as follows:

$$
q_{1}=\left[\begin{array}{ll}
a & b \\
c & d
\end{array}\right], \quad p_{1}=\left[\begin{array}{ll}
p & \tilde{\beta} \\
\beta & \tilde{p}
\end{array}\right] .
$$

Given $H(L)=(1 / 2) \operatorname{tr}\left(L^{2}\right)$, one has $M=R(L)$. A classification of (skew-symmetric with respect to an orthonormal basis in the Lie algebra and constant) $R$-operators obeying the modified Yang-Baxter equations has been obtained by Belavin and Drinfeld for finitedimensional simple Lie algebras $[5,6,7]$. For simplicity, on can use the trivial $R$-operator (or $R$-matrix) for $\operatorname{sl}(4, \mathbb{R}): R(\tilde{h})=0, R\left(E_{\alpha}\right)=(1 / 2) E_{\alpha}$, for any positive root $\alpha, R\left(E_{\alpha}\right)=$ $-1 / 2 E_{\alpha}$, for any negative root $\alpha$, where $\tilde{h}$ is any element of the Cartan subalgebra, and $E_{\alpha}$ is the element corresponding to the root $\alpha$. This $R$-operator satisfies the modified YangBaxter equation $[2,43,47]$ for $c=1 / 4$ :

$$
[R(X), R(Y)]-R\left([X, Y]_{R}\right)+c[X, Y]=0,
$$

where $c$ is a nonvanishing constant, and it corresponds to the triple $\Delta_{1}=0, \Delta_{2}=0, \tau$, of the above-mentioned characterization $[5,6,7]$ of $R$-matrices.

Thus, $L$ leads to

$$
M=\frac{1}{2}\left[\begin{array}{cc}
m & q_{1} \\
-q_{1} & -m
\end{array}\right], \quad \text { where } m=\left[\begin{array}{cc}
0 & \tilde{\beta} \\
-\beta & 0
\end{array}\right] .
$$

The Lax equation $\dot{L}=[L, M]$ implies a system

$$
\dot{p}_{1}=-q_{1}^{2}, \quad\left[m, p_{1}\right]=0, \quad \dot{q}_{1}=\frac{1}{2}\left(q_{1} p_{1}+p_{1} q_{1}\right), \quad\left\{m, q_{1}\right\}=0,
$$

where $\{A, B\}$ stands for $A B+B A$. One finds that $\left[m, p_{1}\right]=0$ and $\left\{m, q_{1}\right\}=0$ can be simultaneously satisfied if either (1) $\beta=\tilde{\beta}=0$, that is, $m=0$, or if (2) $\beta=c=0, p=\tilde{p}$, $a=-d$ (or equivalently $\tilde{\beta}=b=0, p=\tilde{p}, a=-d$ ). For the case (1), one obtains

$$
\begin{gathered}
p=\tilde{p}, \quad \dot{p}=-\left(a^{2}+b c\right), \\
\dot{a}=a p, \quad \dot{b}=b p, \quad \dot{c}=c p .
\end{gathered}
$$


These equations lead to $\dot{p}-p^{2}=C$, where $C$ is a constant of integration. This set of equations is "solvable." Finally, the Lax pair $L, M$ is given by

$$
L=\left[\begin{array}{cccc}
p & 0 & a & b \\
0 & p & c & -a \\
a & b & -p & 0 \\
c & -a & 0 & -p
\end{array}\right], \quad M=R(L)=\frac{1}{2}\left[\begin{array}{cccc}
0 & 0 & a & b \\
0 & 0 & c & -a \\
-a & -b & 0 & 0 \\
-c & a & 0 & 0
\end{array}\right]
$$

with $\operatorname{tr}\left(L^{2}\right)=4\left(p^{2}+a^{2}+b c\right)$. The constraints imposed by the Lax equations have removed certain variables, but still the equations obtained differ from the simple $q_{1} \in$ $\mathrm{GL}(1, \mathbb{R})$ and $p_{1} \in \mathbb{R}$ situation, which corresponds to the nonperiodic Toda model with only 2 particles (see (2.3)). Roughly, the above could be seen as a simple extension via Lax pairs of the $A_{1}$ Toda system. No Hamiltonian interpretation is here presented and a Dirac reduction might be useful, but nonetheless the model is solvable.

For the case (2), one derives a "solvable" system

$$
\dot{p}=-a^{2}, \quad \dot{\alpha}=0, \quad p=\tilde{p}, \quad \dot{a}=a p, \quad \dot{b}=b p,
$$

with the resulting Lax pair

$$
L=\left[\begin{array}{cccc}
p & \alpha & a & b \\
0 & p & 0 & -a \\
a & b & -p & -\alpha \\
0 & -a & 0 & -p
\end{array}\right], \quad M=R(L)=\frac{1}{2}\left[\begin{array}{cccc}
0 & \alpha & a & b \\
0 & 0 & 0 & -a \\
-a & -b & 0 & -\alpha \\
0 & a & 0 & 0
\end{array}\right]
$$

Let us note that still one might wish to derive Poisson submanifold interpretations of the above systems with the Poisson structure associated to the Poisson bracket determined by the $R$-operator, but this is not the intention in this short article. It is thought that nontrivial generalizations could arise for $L$ belonging to $\operatorname{sl}(2 n, \mathbb{R}), n>2$, but more constraint equations would appear. Different $R$-operators could also lead to different extensions, where the classification of Belavin and Drinfeld $[5,6,7]$ can be used. We limit ourselves here with the presentation of first examples, indicating solvable (new) systems, and how they can be derived.

Let us add that the Lax matrix $L$ could belong as well to $\operatorname{gl}(2 n, \mathbb{R})$, and then nontrivial $R$-operators such as those presented in [50] can be used either to generate systems with an $R$-operator related to a linear $r$-matrix (such as the Lax pair with $M$ given by (7.2)), or to provide models via an $R$-operator associated to a Sklyanin structure, as mentioned previously.

For instance, let us consider instead $q_{1}, p_{1} \in \operatorname{gl}(n, \mathbb{R})$, this allows us to have $L \in \operatorname{gl}(2 n$, $\mathbb{R})$, with the simple $R$-operator on the elements of $\mathrm{gl}(2 n, \mathbb{R})$ of the form

$$
R\left(E_{i i}\right)=0, \quad R\left(E_{i j}\right)=\frac{1}{2} E_{i j} \quad \text { when } i<j, \quad R\left(E_{i j}\right)=-\frac{1}{2} E_{i j} \quad \text { when } i>j,
$$

which satisfies the modified Yang-Baxter equation with $c=1 / 4$, and where $i, j=1, \ldots, 2 n$ with the following matrix basis of $\operatorname{gl}(2 n, \mathbb{R}):\left[E_{i j}\right]_{l m}=\delta_{i l} \delta_{j m}$. The Lax equations are then 
2744 On Lax pairs and matrix extended simple Toda systems

written as

$$
\dot{L}=\left[L, R\left(L^{2}\right)\right]
$$

for

$$
H(L)=\frac{1}{2} \operatorname{tr}\left(L^{2}\right), \quad L=\left[\begin{array}{cc}
p_{1} & q_{1} \\
q_{1} & -p_{1}
\end{array}\right] .
$$

They lead to the system $\dot{p}_{1}=\left[p_{1}, \tilde{R}\right], \dot{q}_{1}=\left[q_{1}, \tilde{R}\right]$, with the conditions $\left[q_{1},\left[p_{1}, q_{1}\right]\right]=0$, $\left\{p_{1},\left[p_{1}, q_{1}\right]\right\}=0$, where $\tilde{R}$ denotes the resulting matrix from the action of the $R$-matrix on the $\operatorname{gl}(n, \mathbb{R})$ blocks $q_{1}^{2}+p_{1}^{2}$, of $L^{2}$. The conditions are quite stringent. For example, a solution is $\left[p_{1}, q_{1}\right]=0$, which itself is satisfied when $p_{1}=p 1_{n}$, with $q_{1}$ arbitrary, as for the previous case. But then $\dot{p}_{1}=0$, which forces $p$ to be a constant.

However, the nontrivial $R$-operators of [50] based on a simple triple can be tried for $L \in \operatorname{gl}(2 n, \mathbb{R})$. For $\mathrm{gl}(4, \mathbb{R})$, the linear map obeying the above modified Yang-Baxter equation for $c=1 / 4$ has the form [50]

$$
R\left(E_{i j}\right)=\frac{1}{2}\left[(\theta(j-i)-\theta(i-j)) E_{i j}+2 \delta_{i 1} \delta_{j 2} E_{34}-2 \delta_{i 4} \delta_{j 3} E_{21}+\delta_{i j} \sum_{k=1}^{4} R_{o}^{k i} E_{k k}\right],
$$

where

$$
\left[R_{o}\right]^{i k}=\frac{1}{2}\left[\begin{array}{cccc}
0 & 0 & -1 & 1 \\
0 & 0 & 1 & -1 \\
1 & -1 & 0 & 0 \\
-1 & 1 & 0 & 0
\end{array}\right]
$$

Using the Lax matrix $L$ of (7.15) above, an auxiliary matrix $M=R(L)$ can then be expressed as

$$
M=\frac{1}{2}\left[\begin{array}{cc}
R_{1} & q_{1} \\
-q_{1} & R_{1}
\end{array}\right], \quad \text { with } R_{1}=\left[\begin{array}{cc}
\frac{1}{2}(p-\tilde{p}) & \tilde{\beta} \\
\beta & \frac{1}{2}(\tilde{p}-p)
\end{array}\right]
$$

The Lax equations $\dot{L}=[L, M]$ bring one equation encountered before $\dot{p}_{1}=-q_{1}^{2}$ and the equation $\dot{q}_{1}=1 / 2\left(\left\{p_{1}, q_{1}\right\}+\left[q_{1}, R_{1}\right]\right)$, with a supplementary $\left[q_{1}, R_{1}\right]$ term compared to (6.3), (7.8), but with no initial algebraic constraints, differently from previous examples. Already, these equations are more difficult to solve.

This approach can also be applied to $\operatorname{gl}(2 n, \mathbb{R})$-valued $L$. As mentioned, different $R$ operators can be attempted, either within the linear or quadratic $r$-matrix structures, with more complex resulting differential equations.

\section{Summary and conclusions}

In this short communication, possible noncommutative structures that could provide extensions and deformations of the $A_{1}$ Toda system, a simple integrable model, have 
been explored. At first, toy generalizations of the auxiliary matrix via (non-)commutative functions have been considered. Their quantization could be of interest in future studies. Later, in the spirit of matrices associated with branes coordinates in certain aspects of string theory, some matrix generalizations through a Lax pair preserving the original coordinates splitting have been presented. For instance, $R$-matrix-based terms for the auxiliary matrix have been used to provide examples (some solvable) with (matrix) noncommutativity and deformations. Let us mention that deformations may not be there characterized by a unique parameter such as $\theta$, one can, to a certain extent, see the parameters as related to a set of matrix elements. Studying integrability (and $r$-matrices) by examining Poisson submanifolds would be a possible development, in addition to the consideration of different $R$-matrices in the building of new Lax equations, which can also be associated to other integrable systems. Questions about the description and classification of subspaces of Lax matrices (or operators) leading to nontrivial Lax equations given sets of $R$-operators, and also about Poisson submanifold interpretations can be considered in future works. Let us recall that in the above, the Lax matrices were set to belong to a subspace of $\operatorname{sl}(2 n, \mathbb{R})$ leading to (nonlinear) coupled equations, but different ( $L$-matrices, $R$ ) settings could be examined as well.

\section{Acknowledgment}

This work has been supported in part by the National Sciences and Engineering Research Council (NSERC) of Canada.

\section{References}

[1] H. Aratyn and K. Bering, Compatible Poisson structures of Toda type discrete hierarchy, Internat. J. Modern Phys. A 20 (2005), no. 7, 1367-1388.

[2] O. Babelon, D. Bernard, and M. Talon, Introduction to classical integrable systems, Cambridge Monographs on Mathematical Physics, Cambridge University Press, Cambridge, 2003.

[3] L. M. Baker and D. B. Fairlie, Moyal-Nahm equations, J. Math. Phys. 40 (1999), no. 6, 25392548.

[4] T. Banks, W. Fischler, S. H. Shenker, and L. Susskind, $M$ theory as a matrix model: a conjecture, Phys. Rev. D (3) 55 (1997), no. 8, 5112-5128.

[5] A. A. Belavin and V. G. Drinfel'd, Solutions of the classical Yang-Baxter equation for simple Lie algebras, Funct. Anal. Appl. 16 (1982), 159-180.

[6] - On the classical Young-Baxter equation for simple Lie algebras, Funct. Anal. Appl. 17 (1983), 220-221.

[7] _ Triangle equations and simple Lie algebras, Mathematical Physics Reviews, Vol. 4, Soviet Sci. Rev. Sect. C Math. Phys. Rev., vol. 4, Harwood Academic, Chur, 1984, pp. 93-165.

[8] M. Błaszak and B. M. Szablikowski, Classical R-matrix theory of dispersionless systems. I. $(1+1)$ dimensional theory, J. Phys. A 35 (2002), no. 48, 10325-10344.

[9] I. Cabrera-Carnero and M. Moriconi, Noncommutative integrable field theories in $2 d$, Nuclear Phys. B 673 (2003), no. 3, 437-454.

[10] F. Calogero, Classical Many-Body Problems Amenable to Exact Treatments, Lecture Notes in Physics. New Series m: Monographs, vol. 66, Springer, Berlin, 2001.

[11] P. L. Christiansen, M. F. Jørgensen, and V. B. Kuznetsov, On integrable systems close to the Toda lattice, Lett. Math. Phys. 29 (1993), no. 3, 165-173. 
[12] A. Das and Z. Popowicz, Properties of Moyal-Lax representation, Phys. Lett. B 510 (2001), no. 14, 264-270.

[13] S. de Groot, La Transformation de Weyl et la Fonction de Wigner: Une Forme Alternative de la Mécanique Quantique, Les Presses de l'Université de Montréal, Quebec, 1974.

[14] S. de Groot and L. G. Suttorp, Foundations of Electrodynamics, North-Holland, Amsterdam, 1972.

[15] A. Dimakis and F. Müller-Hoissen, Bicomplexes, integrable models, and noncommutative geometry, Internat. J. Modern Phys. B 14 (2000), no. 22-23, 2455-2460.

[16] , The Korteweg-de-Vries equation on a noncommutative space-time, Phys. Lett. A 278 (2000), no. 3, 139-145.

[17] Noncommutative NLS equation, Czechoslovak J. Phys. 51 (2001), no. 12, 1285-1290.

[18] M. R. Douglas and N. A. Nekrasov, Noncommutative field theory, Rev. Modern Phys. 73 (2001), no. 4, 977-1029.

[19] P. Etingof, I. Gelfand, and V. Retakh, Factorization of differential operators, quasideterminants, and nonabelian Toda field equations, Math. Res. Lett. 4 (1997), no. 2-3, 413-425.

[20] Nonabelian integrable systems, quasideterminants, and Marchenko lemma, Math. Res. Lett. 5 (1998), no. 1-2, 1-12.

[21] H. Flaschka, Discrete and periodic illustrations of some aspects of the inverse method, Dynamical Systems, Theory and Applications (Rencontres, Battelle Res. Inst., Seattle, Wash, 1974) (J. Moser, ed.), Lecture Notes in Phys., vol. 38, Springer, Berlin, 1975, pp. 441-466.

[22] D. J. Gross and N. A. Nekrasov, Solitons in noncommutative gauge theory, J. High Energy Phys. 03 (2001), 044.

[23] M. Hamanaka, Commuting flows and conservation laws for noncommutative Lax hierarchies, J. Math. Phys. 46 (2005), no. 5, 052701, 13 pp.

[24] M. Hamanaka and K. Toda, Noncommutative Burgers equation, J. Phys. A 36 (2003), no. 48, 11981-11998.

[25] E. Kamke, Differentialgleichungen. Lösungsmethoden und Lösungen. Teil II: Partielle Differentialgleichungen erster Ordnung für eine gesuchte Funktion. 4. Aufl., Mathematik und ihre Anwendungen in Physik und Technik. Reihe A., vol. 18, Akademische Verlagsgesellschaft Geest \& Portig K.-G., Leipzig, 1959.

[26] Y. Kodama and J. Ye, Iso-spectral deformations of general matrix and their reductions on Lie algebras, Comm. Math. Phys. 178 (1996), no. 3, 765-788.

[27] I. Krichever and K. L. Vaninsky, The periodic and open Toda lattice, Mirror Symmetry, IV (Montreal, QC, 2000), AMS/IP Stud. Adv. Math., vol. 33, American Mathematical Society, Rhode Island, 2002, pp. 139-158.

[28] B. A. Kupershmidt, Quantizations and integrable systems, Lett. Math. Phys. 20 (1990), no. 1, 19-31.

[29] O. Lechtenfeld, Noncommutative instantons and solitons, Fortschr. Phys. 52 (2004), no. 6-7, 596-605.

[30] O. Lechtenfeld and A. D. Popov, Noncommutative monopoles and Riemann-Hilbert problems, J. High Energy Phys. 01 (2004), 069.

[31] M. Legaré, Reduced systems of $(2,2)$ pseudo-Euclidean noncommutative self-dual Yang-Mills theories, J. Phys. A 35 (2002), no. 26, 5489-5497.

[32] L. C. Li and S. Parmentier, Nonlinear Poisson structures and r-matrices, Comm. Math. Phys. 125 (1989), no. 4, 545-563.

[33] J. Madore, S. Schraml, P. Schupp, and J. Wess, Gauge theory on noncommutative spaces, Eur. Phys. J. C Part. Fields 16 (2000), no. 1, 161-167.

[34] N. A. Nekrasov, Trieste lectures on solitons in noncommutative gauge theories, Superstrings and Related Matters (Trieste, 2000), World Scientific, New Jersey, 2001, pp. 141-205. 
[35] H. Nishino and S. Rajpoot, Noncommutative self-dual supersymmetric Yang-Mills theory, Phys. Lett. B 572 (2003), no. 1-2, 91-100.

[36] M. A. Olshanetsky, Supersymmetric two-dimensional Toda lattice, Comm. Math. Phys. 88 (1983), no. 1, 63-76.

[37] M. A. Olshanetsky and A. M. Perelomov, Classical integrable finite-dimensional systems related to Lie algebras, Phys. Rep. 71 (1981), no. 5, 313-400.

[38] _ Q Quantum integrable systems related to Lie algebras, Phys. Rep. 94 (1983), no. 6, 313 404.

[39] L. D. Paniak, Exact noncommutative KP and KdV multi-solitons, http://arxiv.org/abs/hep-th/ 0105185.

[40] A. D. Popov, A. G. Sergeev, and M. Wolf, Seiberg-Witten monopole equations on noncommutative $\mathbb{R}^{4}$, J. Math. Phys. 44 (2003), no. 10, 4527-4554.

[41] M. Przanowski, J. F. Plebański, and S. Formański, Integrability of SDYM equations for the Moyal bracket Lie algebra, Exact Solutions and Scalar Fields in Gravity: Recent Developments (A. Macías, J. L. Cervantes-Cota, and C. Lämmerzahl, eds.), Kluwer Academic, New York, 2001, pp. 77-87.

[42] Z. Qiao, Generalized $r$-matrix structure and algebro-geometric solution for integrable system, Rev. Math. Phys. 13 (2001), no. 5, 545-586.

[43] A. G. Reyman and M. A. Semenov-Tian-Shansky, Group-theoretical methods in the theory of finite-dimensional integrable systems, Dynamical Systems VII (V. I. Arnold and S. P. Novikov, eds.), Encyclopaedia of Mathematical Sciences, vol. 16, Springer, New York, 1994, pp. 116225.

[44] M. A. Semenov-Tian-Shansky, Quantization of open Toda lattices, Dynamical Systems VII (V. I. Arnold and S. P. Novikov, eds.), Encyclopaedia of Mathematical Sciences, vol. 16, Springer, New York, 1994, pp. 226-259.

[45] V. V. Sokolov and A. V. Tsiganov, Commutative Poisson subalgebras for Sklyanin brackets, and deformations of some known integrable models, Theoret. and Math. Phys. 133 (2002), no. 3, $1730-1743$.

[46] I. A. B. Strachan, The Moyal bracket and the dispersionless limit of the KP hierarchy, J. Phys. A 28 (1995), no. 7, 1967-1975.

[47] Y. B. Suris, The Problem of Integrable Discretization: Hamiltonian Approach, Progress in Mathematics, vol. 219, Birkhäuser, Basel, 2003.

[48] R. J. Szabo, Quantum field theory on noncommutative spaces, Phys. Rep. 378 (2003), no. 4, 207299.

[49] M. Toda, Studies of a non-linear lattice, Phys. Rep. 18 (1975), no. 1, 1-123.

[50] H. Totland, Integrable systems with Belavin-Drinfeld R-matrices, Phys. Lett. A 225 (1997), no. 46, 263-268.

[51] A. V. Tsiganov, Classical R-matrices for generalized so $(p, q)$ tops, http://www.arxiv.org/abs/ nlin.SI/0401025.

[52] R. S. Ward, Integrable and solvable systems, and relations among them, Philos. Trans. Roy. Soc. London Ser. A 315 (1985), no. 1533, 451-457.

[53] E. Witten, Bound states of strings and p-branes, Nuclear Phys. B 460 (1996), no. 2, 335-350.

[54] L. Zhao, W.-Y. Liu, and Z.-Y. Yang, Generalized Toda mechanics associated with classical Lie algebras and their reductions, Commun. Theor. Phys. (Beijing) 41 (2004), no. 3, 339-348.

M. Legaré: Department of Mathematical Sciences, University of Alberta, Edmonton, AB, Canada T6G 2G1

E-mail address: mlegare@math.ualberta.ca 


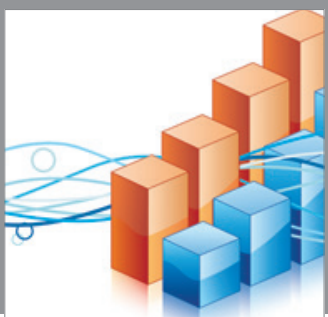

Advances in

Operations Research

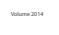

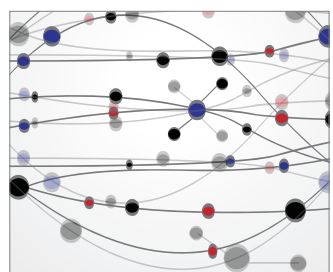

\section{The Scientific} World Journal
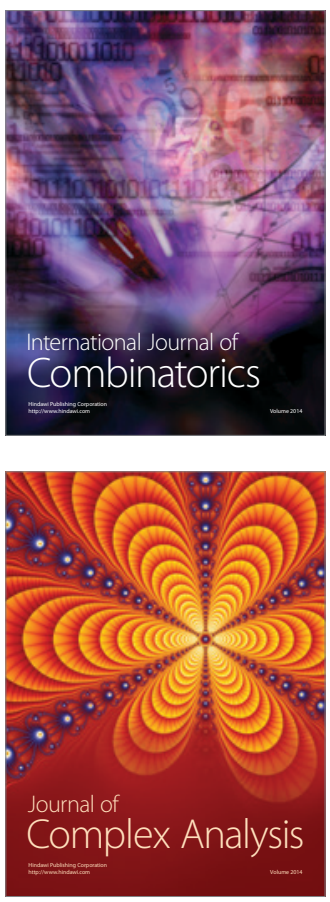

International Journal of

Mathematics and

Mathematical

Sciences
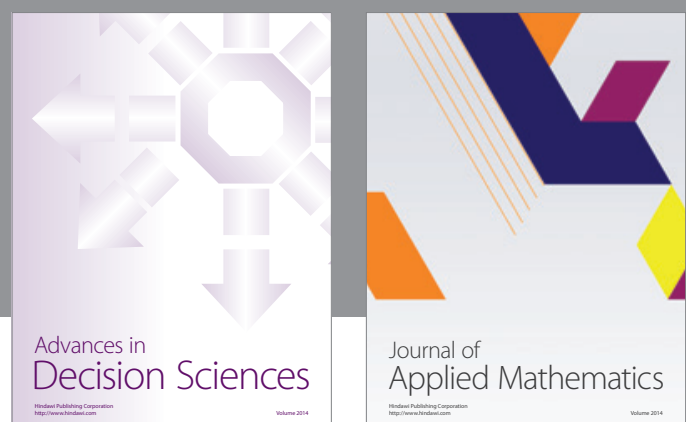

Journal of

Applied Mathematics
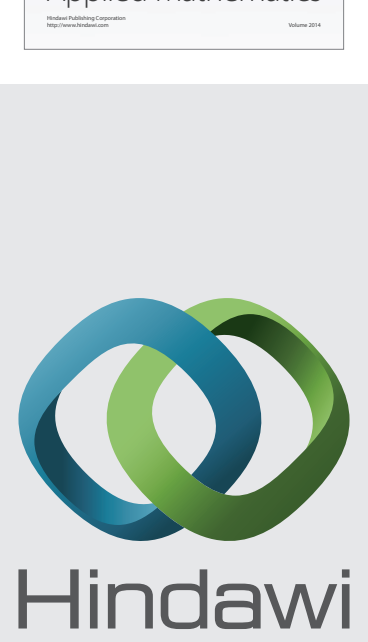

Submit your manuscripts at http://www.hindawi.com
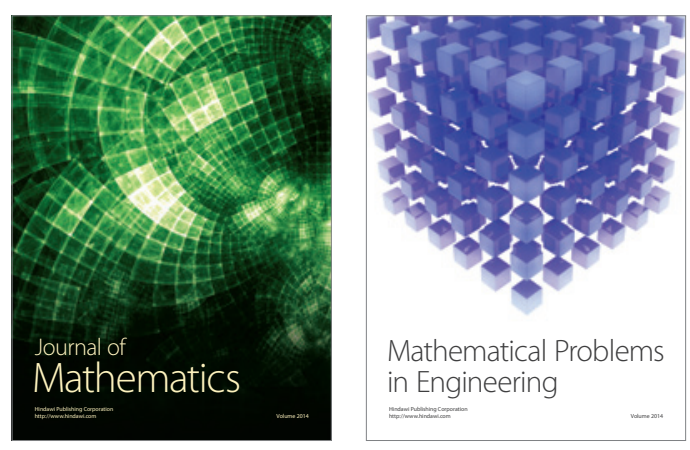

Mathematical Problems in Engineering
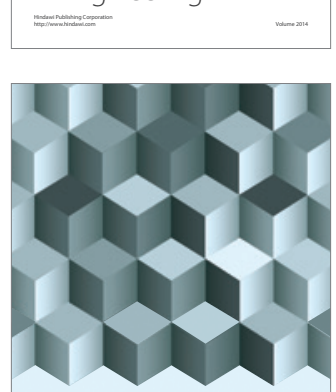

Journal of

Function Spaces
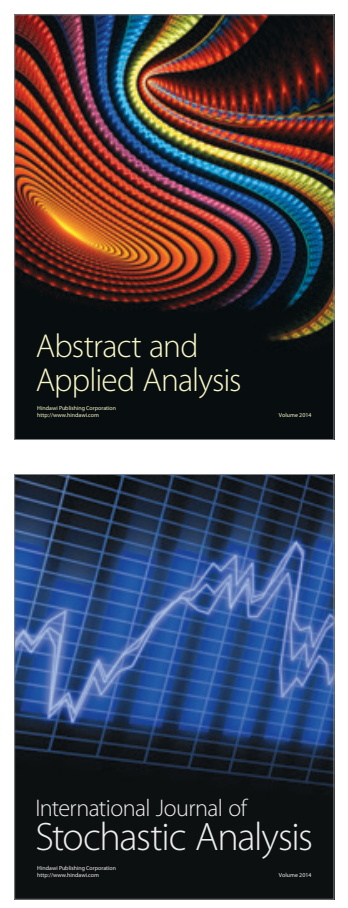

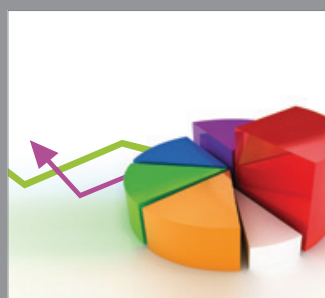

ournal of

Probability and Statistics

Promensencen
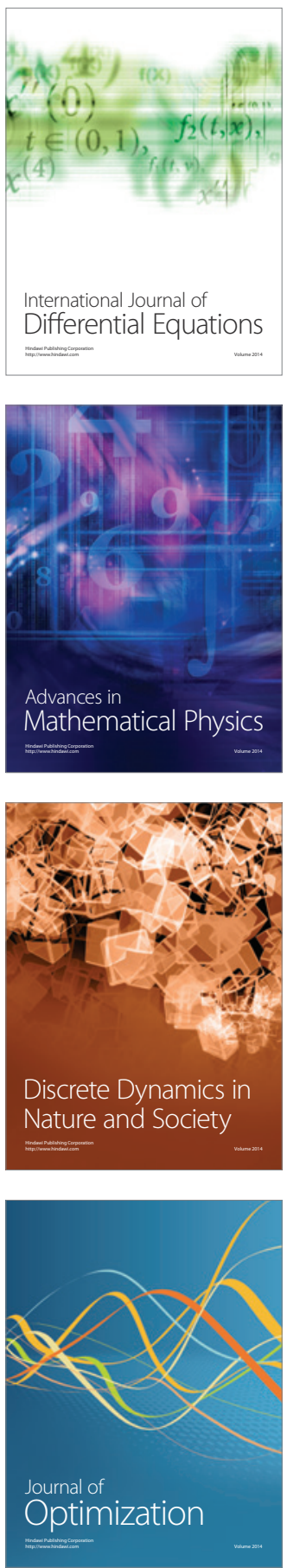